\title{
RESEARCH ON THE STATISTICAL CHARACTERISTICS OF CROSSTALK IN NAVAL SHIPS WIRING HARNESS BASED ON POLYNOMIAL CHAOS EXPANSION METHOD
}

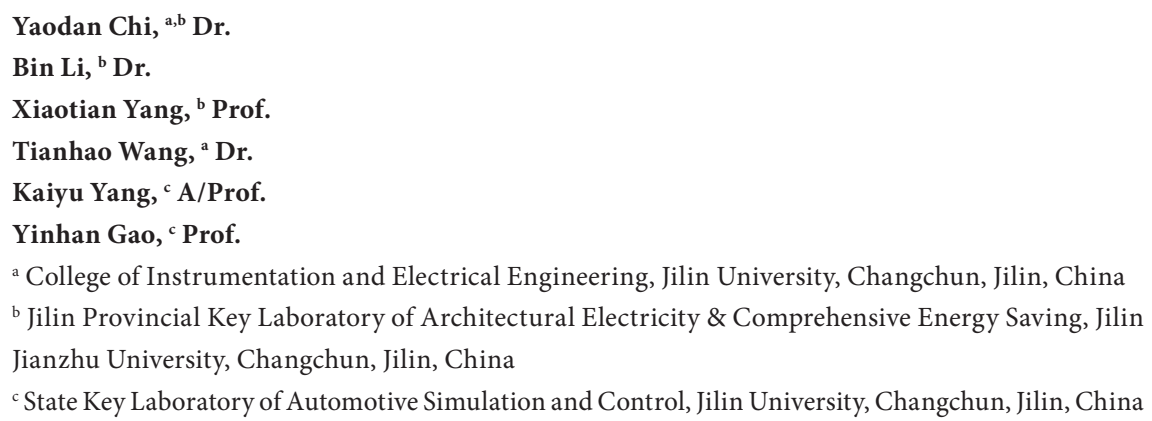

\begin{abstract}
Crosstalk in wiring harness has been studied extensively for its importance in the naval ships electromagnetic compatibility field. An effective and high-efficiency method is proposed in this paper for analyzing Statistical Characteristics of crosstalk in wiring harness with random variation of position based on Polynomial Chaos Expansion (PCE). A typical 14-cable wiring harness was simulated as the object of research. Distance among interfering cable, affected cable and GND is synthesized and analyzed in both frequency domain and time domain. The model of naval ships wiring harness distribution parameter was established by utilizing Legendre orthogonal polynomials as basis functions along with prediction model of statistical characters. Detailed mean value, mean square error, probability density function and reasonable varying range of crosstalk in naval ships wiring harness are described in both time domain and frequency domain. Numerical experiment proves that the method proposed in this paper, not only has good consistency with the MC method can be applied in the naval ships EMC research field to provide theoretical support for guaranteeing safety, but also has better time-efficiency than the MC method. Therefore, the Polynomial Chaos Expansion method.
\end{abstract}

Keywords: Naval ships wiring harness; Crosstalk; Polynomial chaos expansion; Statistical characteristics

\section{INTRODUCTION}

Crosstalk in naval ships wiring harness can be determined by numerous reasons, such as the randomness of binding and fixation, variety of driving speed, jolt and vibration caused by operating condition etc. [1-3]. Research revealed that the crosstalk in naval ships wiring harness is very sensitive to positions. Vibration of the crosstalk is able to be raised up to $20 \mathrm{~dB}$ by the change of relative positions. However, computational electromagnetics methods such as multiconductor transmission line theory (MTL), finite difference time domain (FDTD) and method of moment (MOM) are mainly focus on the assumption that all cable positions are fixed and invariable [4-9]. Calculations and analysis are based on this assumption. Compute resources can be consumed tremendously and results can be dissatisfaction if these methods are adopted to calculation of random placed cable. Therefore, it is becoming a trend to research the fast and reasonable method for analyzing the statistical characteristics of crosstalk in wiring harness in recent years.

Monte Carlo (MC) method is an important method that are applied in this field [10, 11]. Generally, MC method along with numerous methods such as convolution method and collocation method are adopted to analyze the statistical characters of wiring harness. Statistical characters including the mean value, mean square error and reasonable varying range of naval ships wiring harness are normally calculated 
besides probability density function for analysis [12]. However, the calculating efficiency is influenced by generating crosssection samples when these methods are adopted to predict the related interference statistical characters. In this way, it is not efficiency to use these methods in actual industrial applications.

In this paper, a polynomial chaos expansion (PCE) method is proposed to analysis statistical characters including the mean value, mean square error, reasonable varying range and probability density function in both time domain and frequency domain for naval ships wiring harness. This method is based on the distribution type and varying position of naval ships cable to choose orthogonal polynomial basis function. Then, chaos polynomial expansion of distributed parameter is performed. Meanwhile, corresponding coefficient of each expansion term is obtained. In this process, related statistical characters of naval ships cable distribution parameter can be determined by the property of chaos polynomial expansion. Then, combined with boundary conditions, related statistical characters of crosstalk in naval ships wiring harness in frequency domain can be determined by chaos polynomial expansion. Finally, results of PCE and MC method are compared and discussed. To be noticed that the geometric position variable of cables is based on Gaussian distribution and uniform distribution, and the uniform distribution is discussed specially in this paper. The method proposed in this paper can be used as an effective tool to analysis electromagnetic compatibility in naval ships aspect.

\section{THEORY}

Polynomial chaos expansion method is a powerful and effective mehtod which is widely applied in numerous aspects such as industrial, material research and mathematical field [13-18]. The fundamentals of polynomial chaos expansion and uniform distribution will be briefly summarized here only to guide the audients and define units. Besides Gaussian distribution, uniform distribution is also an important type of distribution which is adopted in the engineering field [1921]. It exerts its influence in the statistical aspect. Thus, in order to analysis its statistical characters, polynomial chaos expansion will be performed for random event with random variable in accordance with uniform distribution. Under such circumstances, the corresponding basis functions are Legendre orthogonal polynomials. In this way, assume a random process is expressed as:

$$
Y=y(\xi)
$$

The random variable $\boldsymbol{\xi}=\left[\xi_{1}, \xi_{2}, \ldots, \xi_{n}\right]^{T}$ can be seen as n-dimensional independent uniform distribution input. The expression of polynomial chaos with Legendre orthogonal polynomials as basis functions can be shown as:

$$
\begin{aligned}
Y & =b_{0} L_{0} \\
& +\sum_{i=1}^{n} b_{i} L_{1}\left(\xi_{i}\right) \\
& +\sum_{i=1}^{n} \sum_{j=1}^{i} b_{i j} L_{2}\left(\xi_{i}, \xi_{j}\right) \\
& +\sum_{i=1}^{n} \sum_{j=1}^{i} \sum_{k=1}^{j} b_{i j k} L_{3}\left(\xi_{i}, \xi_{j}, \xi_{k}\right) \\
& +\cdots
\end{aligned}
$$

where $b_{0} 、 b_{i} 、 b_{i j}, b_{i j k} \cdots \cdots$ are certain coefficient to be solved and $L_{p}$ is a p-order Legendre orthogonal polynomial. For a p-order Legendre orthogonal polynomial at $\mathrm{n}$-dimension, it can be expressed as follow:

$$
L_{p}(\zeta)=\frac{1}{2^{n} n !} \frac{\partial^{p}}{\partial \xi_{i} \cdots \partial \xi_{j}}\left\{\left(\zeta^{2}-1\right)^{p}\right\}
$$

Similarly, expression can be obtained in equation (4) with $\mathrm{m}$-term $\mathrm{p}$-order finite terms by using the truncation of equation (2).

$$
Y=\sum_{i=0}^{m-1} \hat{c}_{i} \varphi_{i}(\xi)
$$

Then the certain coefficient $\hat{c}_{i}$ to be solved is corresponding to $b_{0} 、 b_{i} 、 b_{i j} 、 b_{i j k} \ldots . .$. and Legendre orthogonal polynomials are corresponding to the polynomials obtained by equation (3). In addition, the solution of Legendre orthogonal polynomials is a set of complete orthogonal basis functions that satisfy the below equation:

$$
\left\langle\varphi_{i}, \varphi_{j}\right\rangle=\left\langle\varphi_{i}^{2}\right\rangle \delta_{i j}
$$

where $\delta_{i j}$ is Kronecker delta function that can be expressed as:

$$
\delta_{i j} \begin{cases}0 & i=j \\ 1 & i \neq j\end{cases}
$$

For Legendre orthogonal polynomials, weighting function $W(\boldsymbol{\xi})$ is expressed as:

$$
W(\boldsymbol{\xi})= \begin{cases}\frac{1}{2^{n}} & |\boldsymbol{\xi}| \leq 1 \\ 0 & |\boldsymbol{\xi}|>1\end{cases}
$$

Similarly, coefficient $\hat{c}_{i}$ and term $\mathrm{m}$ can be obtained by using equation (8) and (9).

$$
\mathrm{m}=\frac{(p+n) !}{p ! n !}
$$




$$
\begin{aligned}
Y_{\text {Hmean }} & =E_{P C}(Y(\xi)) \\
& =\int_{D_{\xi}} Y(\xi) W(\xi) \mathrm{d} \xi \\
& =\int_{D_{\xi}}\left(\sum_{i=0}^{m-1} \hat{c}_{i} \varphi_{i}(\xi)\right) W(\xi) \mathrm{d} \xi \\
& =\hat{c}_{0} \int_{D_{\xi}} \varphi_{0}(\xi) W(\xi) \mathrm{d} \xi+\sum_{i=1}^{m-1} \hat{c}_{i} \int_{D_{\xi}} \varphi_{i}(\xi) W(\xi) \mathrm{d} \xi \\
& =\hat{c}_{0}+\sum_{i=1}^{m-1} \hat{c}_{i} \int_{D_{\xi}} \varphi_{0}(\xi) \varphi_{i}(\xi) W(\xi) \mathrm{d} \xi \\
& =\hat{c}_{0}
\end{aligned}
$$

Be coupled with equation (9) and (10), the mean response $Y_{\text {Lmean }}$ and variance $Y_{\text {Lvar }}$ based on the random events of chaos unfolding Legendre orthogonal polynomials can be expressed below in equation (11) and (12):

$$
\begin{aligned}
Y_{\text {Hvar }} & =\operatorname{Var}_{P C}(Y(\xi)) \\
& =E_{P C}\left[\left(Y(\xi)-Y_{\text {mean }}(\xi)\right)^{2}\right] \\
& =\int_{D_{\xi}}\left(Y(\xi)-Y_{\text {mean }}(\xi)\right)^{2} W(\xi) \mathrm{d} \xi \\
& =\int_{D_{\xi}}\left(Y(\xi)-\hat{c}_{0}\right)^{2} W(\xi) \mathrm{d} \xi \\
& =\int_{D_{\xi}}\left(\sum_{i=1}^{m-1} \hat{c}_{i} \varphi_{i}(\xi)\right)^{2} W(\xi) \mathrm{d} \xi \\
& =\sum_{i=1}^{m-1} \sum_{j=1}^{m-1} \int_{D_{\xi}} \hat{c}_{i} \varphi_{i}(\xi) \hat{c}_{i} \varphi_{j}(\xi) W(\xi) \mathrm{d} \xi \\
& =\sum_{i=1}^{m-1}\left[\hat{c}_{i}^{2}\left\langle\varphi_{i}^{2}\right\rangle\right] \\
& Y_{\text {Lmean }}=\hat{c}_{0} \\
& Y_{\text {Lvar }}=\sum_{i=1}^{m-1}\left[\hat{c}_{i}^{2}\left\langle\varphi_{i}^{2}\right\rangle\right]
\end{aligned}
$$

Therefore, the method of performing polynomial chaos expansion to random events, which own random variables obey uniform distribution, is obtained and is adopted in this paper.

\section{SIMULATION AND ANALYSIS}

\section{MODELLING OF NAVAL SHIPS WIRING HARNESS}

In order to investigate the crosstalk of naval ships wiring harness accurately and reasonably, it is essential to quantify key factors and to establish the model. Therefore, the method of performing polynomial chaos expansion is adopted to analyze the statistical character of distribution parameter and cable interference.

The cross section of a typical naval ships wiring harness, which is according to the industrial standard, is shown in Fig. 1. It contains 14 independent copper cables that are fixed tightly inside the insulating layer made of polyvinyl chloride (PVC). The radius of the wiring harness and a single cable is $4.5 \mathrm{~mm}$ and $0.4 \mathrm{~mm}$, respectively. The distance between central position and the reference ground (GND) is $25 \mathrm{~mm}$. Assume the relative dielectric constant $\varepsilon_{\mathrm{r}}=3.0$, the thickness of insulating layer is $0.6 \mathrm{~mm}$, the length of the wiring harness is $1.25 \mathrm{~m}$ and the weak-coupling cables are electrically lossless.

The equivalent circuit model of the interfering cable, the affected cable and GND is shown in Fig. 2. Considering that there are many cables in the wiring harness, the position range is relatively large and the insulating layer is thick, the inductance and capacitance matrix per unit are obtained that is convenient for polynomial chaos expansion.
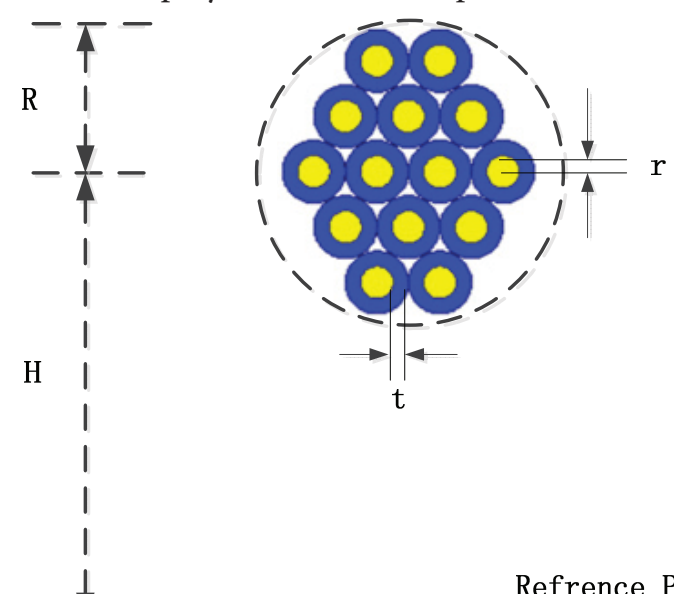

Fig. 1 Modeling of wiring harness cross section

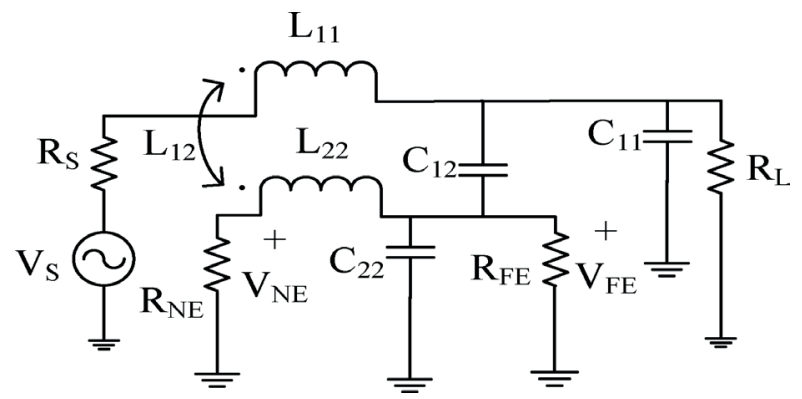

Fig. 2 Equivalent circuit model of the interfering cable, the affected cable and GND

The inductance matrix per unit $\mathbf{L}$ can be expressed as:

$$
\mathbf{L}=\left[\begin{array}{ll}
\mathrm{L}_{11} & \mathrm{~L}_{12} \\
\mathrm{~L}_{21} & \mathrm{~L}_{22}
\end{array}\right]=\left[\begin{array}{cc}
\frac{\mu_{0}}{2 \pi} \ln \left(\frac{2 h_{1}}{r_{1}}\right) & \frac{\mu_{0}}{4 \pi} \ln \left(1+4 \frac{h_{1} h_{2}}{d^{2}}\right) \\
\frac{\mu_{0}}{4 \pi} \ln \left(1+4 \frac{h_{1} h_{2}}{d^{2}}\right) & \frac{\mu_{0}}{2 \pi} \ln \left(\frac{2 h_{2}}{r_{2}}\right)
\end{array}\right]
$$

where $\mu_{0}$ is permeability of vacuum, $r_{1}$ and $r_{2}$ are the radius of the interfering cable and the affected cable respectively, 
$h_{1}, h_{2}$ and $d$ are the distance to GND and relative distance of the interfering cable and the affected cable, respectively.

The inductance matrix per unit $\mathbf{L}$ can be ignored considering that there are insolating layers. However, the capacitance matrix per unit $\mathbf{C}$ is affected and can be expressed as:

$$
\begin{aligned}
\mathbf{C} & =\left[\begin{array}{cc}
\mathrm{C}_{11}+\mathrm{C}_{12} & -\mathrm{C}_{12} \\
-\mathrm{C}_{21} & \mathrm{C}_{22}+\mathrm{C}_{12}
\end{array}\right] \\
& =\left[\begin{array}{cc}
\frac{1}{2 \pi \varepsilon_{0}}\left(\frac{1}{\varepsilon_{r}} \ln \frac{1}{r_{1}}+\varepsilon_{e} \ln \frac{1}{r_{1}+t_{1}}-\ln \frac{1}{2 h_{1}}\right) & \frac{1}{4 \pi \varepsilon_{0}} \ln \left(1+\frac{4 h_{1} h_{2}}{d^{2}}\right) \\
\frac{1}{4 \pi \varepsilon_{0}} \ln \left(1+\frac{4 h_{1} h_{2}}{d^{2}}\right) & \frac{1}{2 \pi \varepsilon_{0}}\left(\frac{1}{\varepsilon_{r}} \ln \frac{1}{r_{2}}+\varepsilon_{e} \ln \frac{1}{r_{2}+t_{2}}-\ln \frac{1}{2 h_{2}}\right)
\end{array}\right]^{-1}
\end{aligned}
$$

where $\varepsilon_{e}=\left(\varepsilon_{r}-1\right) / \varepsilon_{r}$ is effective dielectric constant, $\varepsilon_{0}$ is permittivity of vacuum and $\varepsilon_{r}$ is relative dielectric constant, $t_{1}$ and $t_{2}$ are the thickness of the interfering cable and the affected cable, respectively.

From equation (13) and (14), the value of each element of distribution parameter matrix is decided by cable positions when the wiring harness type is fixed. These positions are consisting of distance $h_{1}$ between the interfering cable and GND, distance $h_{2}$ between the affected cable and GND and the relative distance $d$. In this way, the statistical character of distribution parameter and cable interference can be determined by above three coefficients. Therefore, the polynomial chaos expansion method is performed in the following sections to investigate the statistical characters under the condition of uniform distribution.

\section{STATISTICAL ANALYSIS OF CROSSTALK IN WIRING HARNESS BASED ON PCE METHOD}

The elements of inductance and capacitance augmented matrix per unit can obtained based on PCE method. Substitute the results obtained from equation 3 and 15 based on the Legendre orthogonal basis transfer function $\varphi_{i}$ and the corresponding coefficient $\mathbf{L}_{i}$ and $\mathbf{C}_{i}$ into equation 16, while $\mathrm{p}=1$. Then, substitute the generated normal random number which is comply with the requirement of parameter into equation 16.

$$
\begin{gathered}
\hat{\mathcal{c}}_{(a, b), i}=\int_{R^{n}} \frac{\hat{\mathcal{c}}_{(a, b)}(\boldsymbol{\xi}) \phi_{i}(\boldsymbol{\xi})}{\sqrt{(2 \pi)^{n}}} \exp \left(-\frac{1}{2} \xi^{\mathrm{T}} \boldsymbol{\xi}\right) /\left\langle\varphi_{i}^{2}\right\rangle \\
\mathbf{L}=\sum_{i=0}^{m-1} \mathbf{L}_{i} \varphi_{i}(\boldsymbol{\xi}) \mathbf{C}=\sum_{i=0}^{m-1} \mathbf{C}_{i} \varphi_{i}(\boldsymbol{\xi})
\end{gathered}
$$

Based on the PCE method, the crosstalk of the model shown is analyzed both in time domain and frequency domain. Assume $h_{1}, h_{2} \in[20.5,29.5] \mathrm{mm}, d \in[1.2,7.8]$ $\mathrm{mm}$, the following expression can be obtained.

$$
\left\{\begin{array}{l}
h_{1}=4.5 \xi_{1}+25 \\
h_{2}=4.5 \xi_{2}+25 \\
d=3.3 \xi_{3}+4.5
\end{array}\right.
$$

For $\boldsymbol{\xi}=\left[\xi_{1}, \xi_{2}, \xi_{3}\right]^{\mathrm{T}}, \mathrm{p}=1, \mathrm{~m}=4$, the following equation can be obtained:

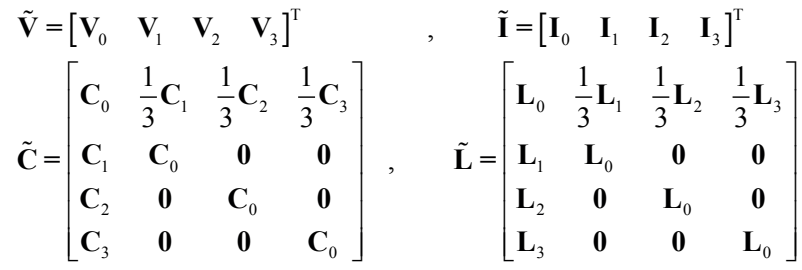

where the input of the 3-dimention random variable is in the range $[-1,1], p$ is the order of expansion and $m$ is the number of terms. Assume excitation voltage source is a single frequency sine wave signal and the amplitude is $1 \mathrm{~V}$. The frequency range is from $1 \mathrm{kHz}$ to $300 \mathrm{MHz}$. Under two conditions of terminating high impedance and low impedance, the mean value, mean square error and reasonable varying range of the crosstalk in near-end can be calculated based on the Legendre-PCE method. Results are compared with MC results and are shown in Fig. 3 and Fig. 4.

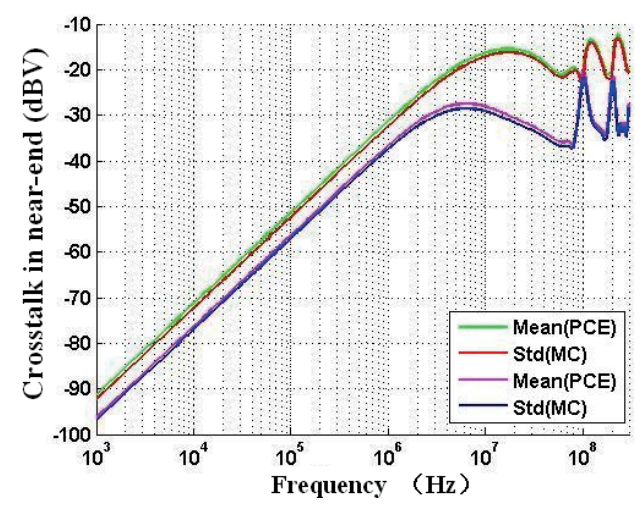

(a) Mean value and mean square error

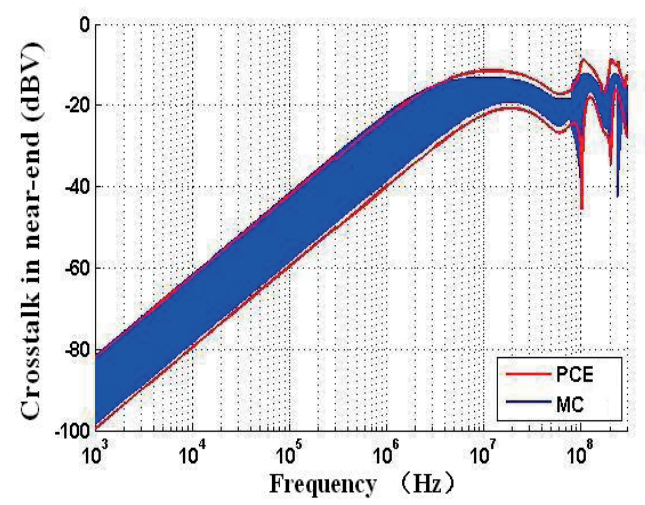

(b) Reasonable varying range

Fig. 3 Comparison of crosstalk in near-end based on Legendre-PCE and MC method in frequency domain while terminating high impedance 


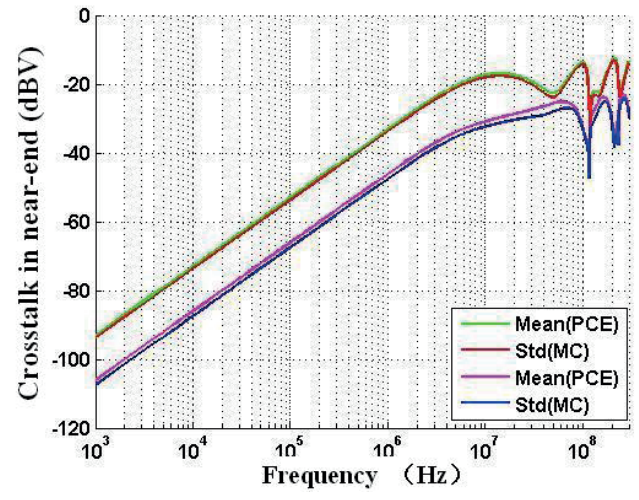

(a) Mean value and mean square error

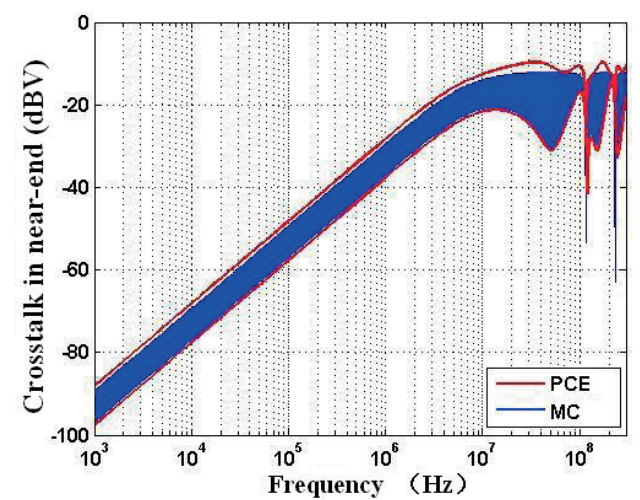

(b) Reasonable varying range

Fig. 4 Comparison of crosstalk in near-end based on Legendre-PCE and MC method in frequency domain while terminating low impedance

Similarly, Legendre-PCE method is adopted to investigate the mean value, mean square error and reasonable varying range of the crosstalk in far-end of naval ships wiring harness for both terminating high and low impedance in frequency domain. Results are shown in Fig. 5 and 6 and are compared with MC results of 3000 samples.

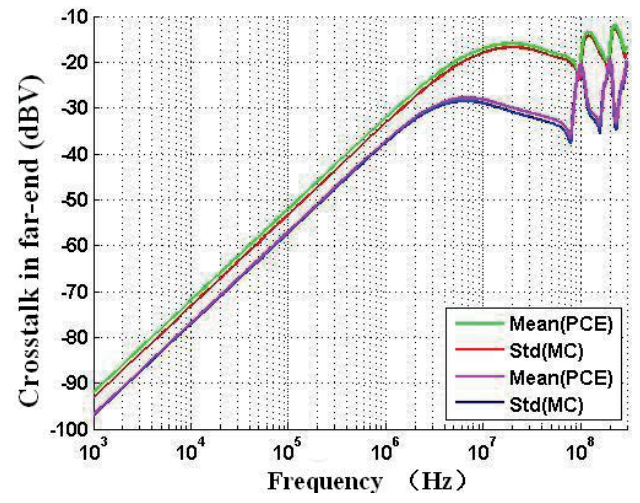

(a) Mean value and mean square error

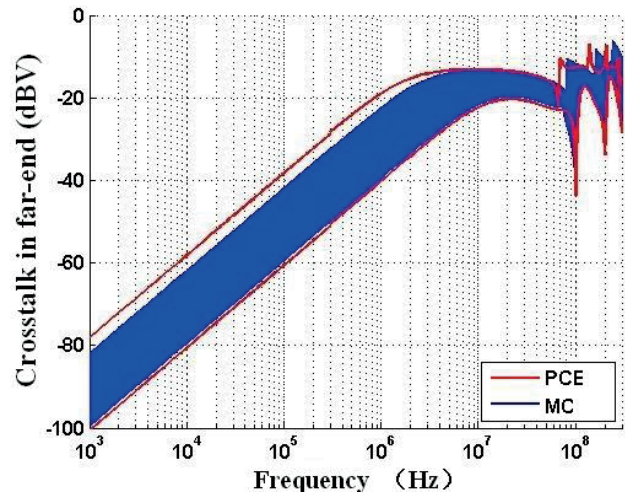

(b) Reasonable varying range

Fig. 5 Comparison of crosstalk in far-end based on Legendre-PCE and MC method in frequency domain while terminating high impedance

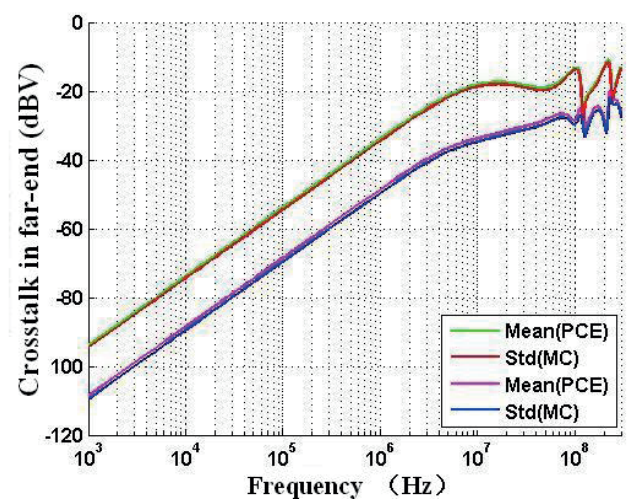

(a) Mean value and mean square error

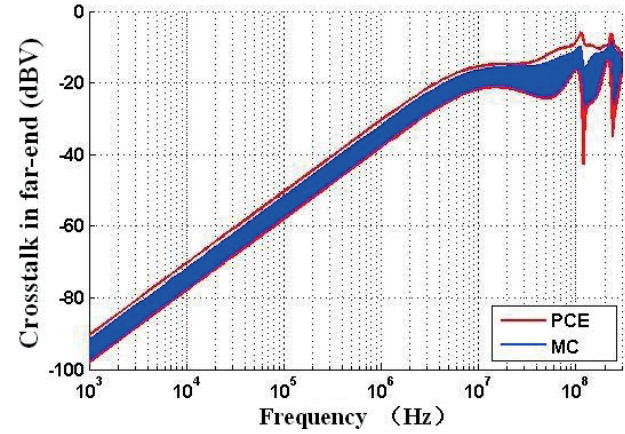

(b) Reasonable varying range

Fig. 6 Comparison of crosstalk in far-end based on Legendre-PCE and MC method in frequency domain while terminating low impedance

Then, $\mathbf{V}_{N(F) E i}$ can be obtained by the following equations. And probability density function of crosstalk both in nearend and far-end based on Legendre-PCE and MC method in frequency domain can be determined by using equation 21 at specific frequency. Considering two conditions that $\mathrm{f}=250 \mathrm{kHz}$ and $\mathrm{f}=300 \mathrm{MHz}$, results are obtained by using 3000 samples and are compared with MC results which are shown in Fig. 7 and Fig. 8.

$$
\frac{\mathrm{d}}{\mathrm{d} z} \tilde{\mathbf{V}}(z, s)=-\tilde{\mathbf{C}} \tilde{\mathbf{I}}(z, s)
$$




$$
\begin{gathered}
\frac{\mathrm{d}}{\mathrm{d} z} \tilde{\mathbf{I}}(z, s)=-\tilde{\mathbf{L}} \tilde{\mathbf{V}}(z, s) \\
\left|\mathbf{V}_{N(F) E}(s)\right|=\left|\sum_{i=0}^{m-1} \mathbf{V}_{N(F) E i}(s) \varphi_{i}(\boldsymbol{\xi})\right|
\end{gathered}
$$
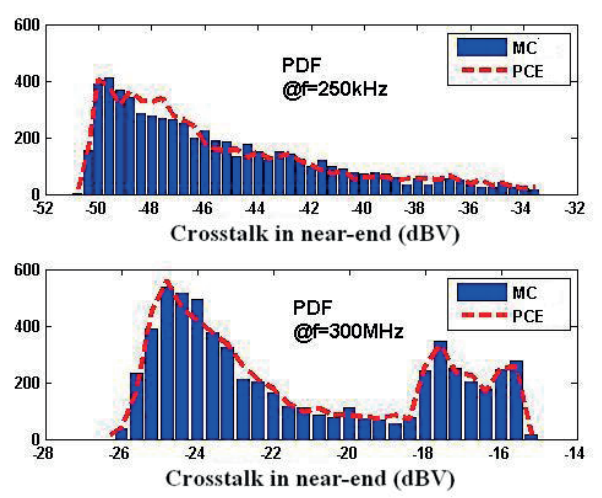

(a) Terminating high impedance
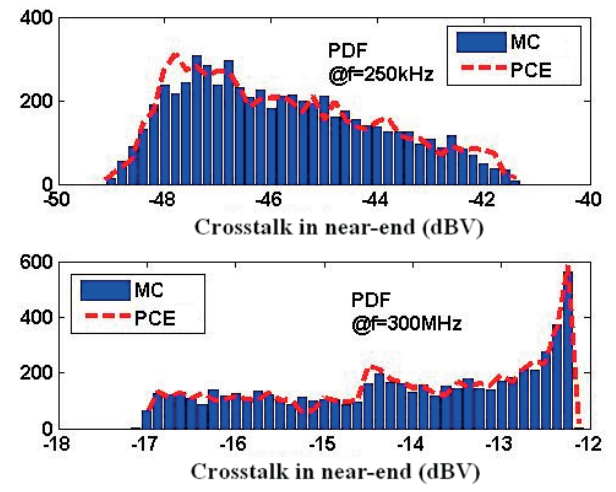

(b) Terminating low impedance

Fig. 7 Comparison of probability density function of crosstalk in near-end based on Legendre-PCE and MC method in frequency domain
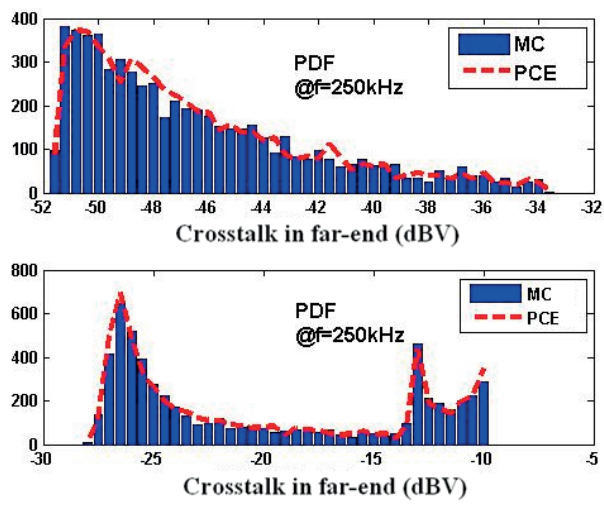

(a) Terminating high impedance
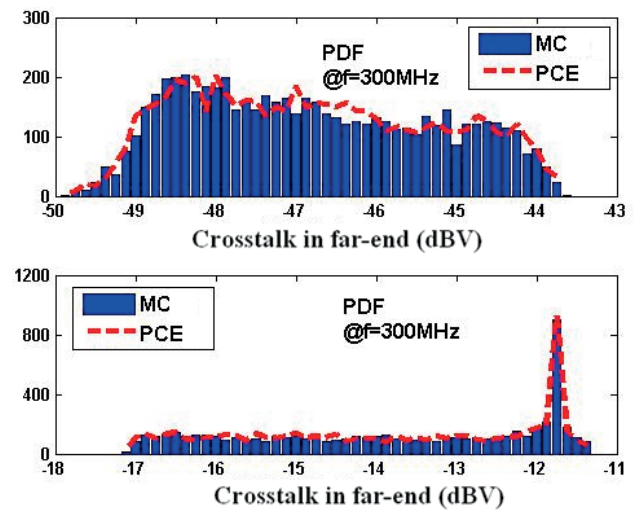

(b) Terminating low impedance

Fig. 8 Comparison of probability density function of crosstalk in far-end based on Legendre-PCE and MC method in frequency domain

Experiments from Fig. 3 to Fig. 8 show that the LegendrePCE method has good coincidence with the MC method for calculating the mean value and mean square error of crosstalk in naval ships wiring harness in frequency domain. To be noticed that the distance between interfering cable and GND, distance between the affected cable and GND and the relative distance confirm to uniform distribution. And the sampling number is 3000 for each experiment. Meanwhile, the probability density function of crosstalk obtained by Legendre-PCE is also close to the MC results.

Then, analysis of statistical characters of crosstalk in naval ships wiring harness in time domain is performed. Crosstalk in near-end and far-end in time domain can be expressed as:

$$
v_{N(F) E}(t)=\sum_{i=0}^{m-1} v_{N(F) E i}(t) \varphi_{i}(\xi)
$$

where $v_{N(F) E i}(t)$ can be obtained by using the equation below:

$$
\begin{gathered}
v_{N(F) E i}(t)=\hat{\mathrm{H}}(0) \mathbf{V}_{N(F) E i}(0)+\sum_{n=1}^{\infty}\left(2\left|\mathrm{H}\left(j n \omega_{0}\right)\right|\left|\mathbf{V}_{N(F) E i}\left(j n \omega_{0}\right)\right| *\right. \\
\cos \left(n \omega_{0} t+\angle \hat{\mathrm{H}}\left(j n \omega_{0}\right)+\angle \mathbf{V}_{N(F) E i}\left(j n \omega_{0}\right)\right)
\end{gathered}
$$

In Equation (23), $\mathrm{i}=0,1, \cdots$ and $\mathrm{m}-1, \mathbf{V}_{N(F) E i}(s)$ can be calculated by using Equations (19) and (20). According to the property of orthogonal polynomial chaotic expansion, the mean value and mean square error of crosstalk in near-end and far-end in time domain can be determined by Equation (24) and (25), similar to the frequency domain, the reasonable varying range is set as

$$
\begin{gathered}
{\left[\bar{v}_{N(F) E}(t)-3 \sigma_{v_{N(F) E}}(t), \bar{v}_{N(F) E}(t)+\sigma_{v_{N(F) E}}(t)\right] .} \\
\bar{v}_{N(F) E}(t)=v_{N(F) E 0}(t)
\end{gathered}
$$




$$
\sigma_{v_{N(F) E}}(t)=\sqrt{\sum_{i=1}^{m} v_{N(F) E i}^{2}(t)\left\langle\varphi_{i}^{2}\right\rangle}
$$

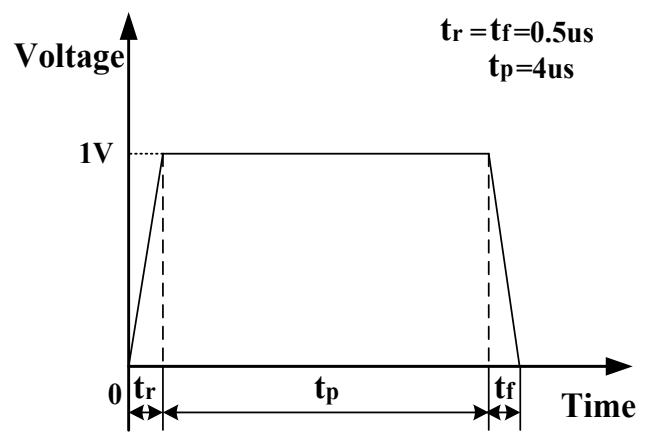

Fig. 9 The excitation voltage source in time domain

Similar to the frequency domain, mean value, mean square error and reasonable varying range of crosstalk in near-end are determined while both terminating high and low impedance. The excitation voltage source is shown in Fig. 9. Results calculated by Legendre-PCE and MC are compared and are shown in Fig. 10 and Fig. 11.. The sampling number of MC method is 3000 for each experiment.
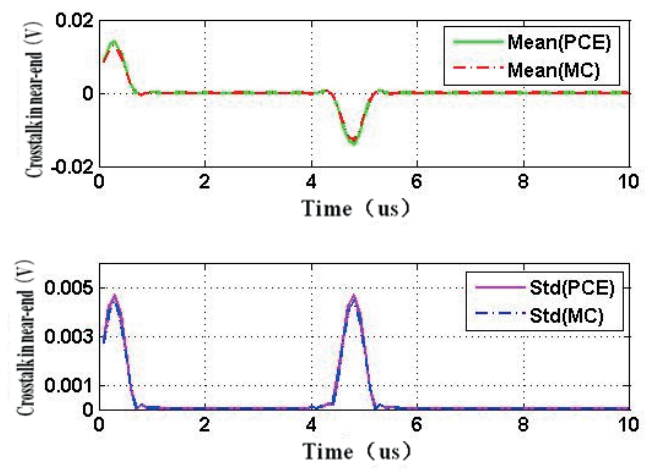

(a) Mean value and mean square error

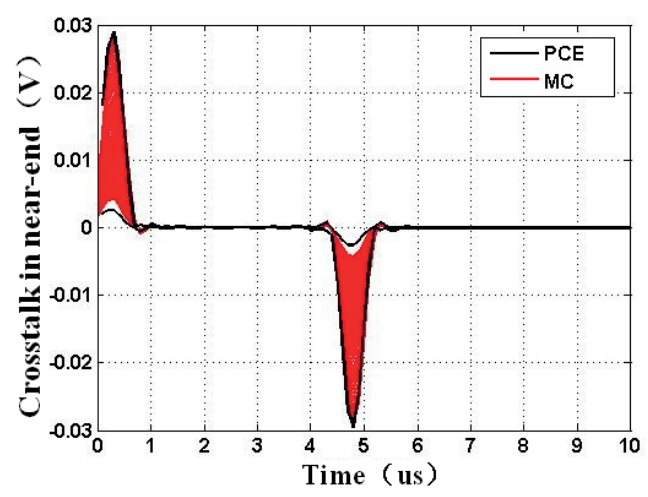

(b) Reasonable varying range

Fig. 10 Comparison of crosstalk in near-end based on Legendre-PCE and MC method in time domain while terminating high impedance
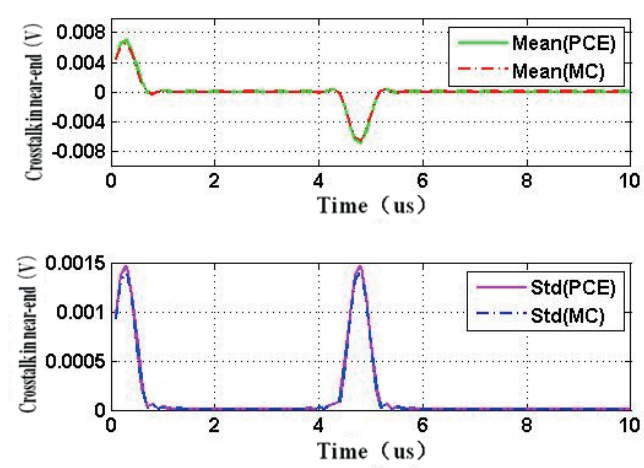

(a) Mean value and mean square error

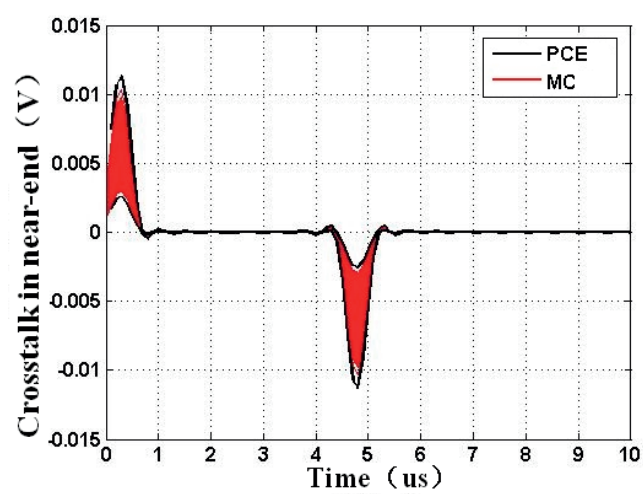

(b) Reasonable varying range

Fig. 11 Comparison of crosstalk in near-end based on Legendre-PCE and MC method in time domain while terminating low impedance

Refer to the above analyzing method, numerical experiment is carried out to investigate the crosstalk of affected cable while terminating high and low impedance in time domain. Results are plotted and compared in Fig. 12 and Fig. 13.
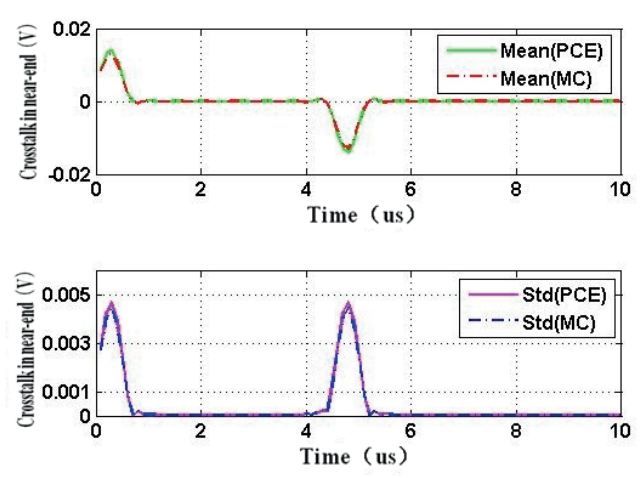

(a) Mean value and mean square error 


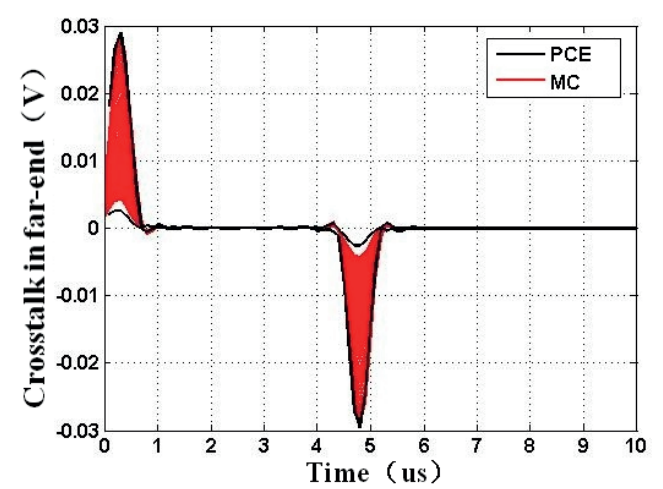

(b) Reasonable varying range

Fig. 12 Comparison of crosstalk in far-ene based on Legendre-PCE and MC method in time domain while terminating high impedance
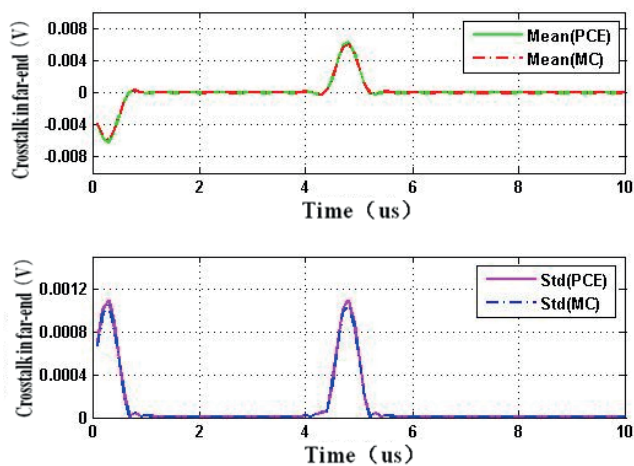

(a) Mean value and mean square error

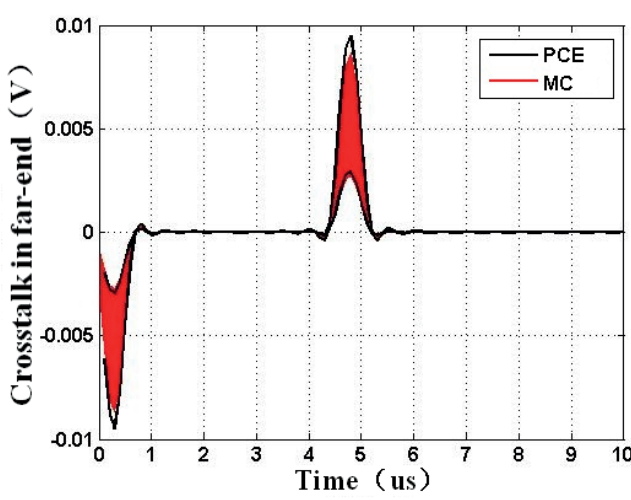

(b) Reasonable varying range

Fig. 13 Comparison of crosstalk in far-end based on Legendre-PCE and MC method in time domain while terminating low impedance

Finally, the probability density function of naval ships wiring harness is shown in Fig. 14 and Fig. 15.
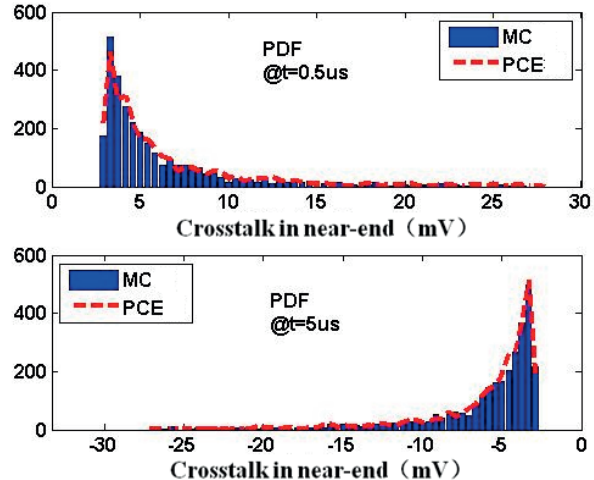

(a) Terminating high impedance

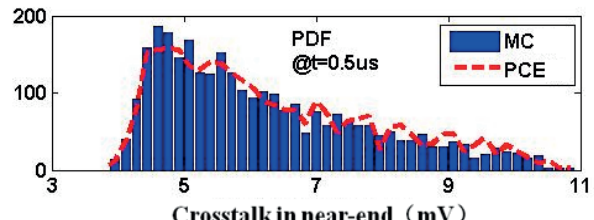

Crosstalkin near-end $(\mathrm{mV})$

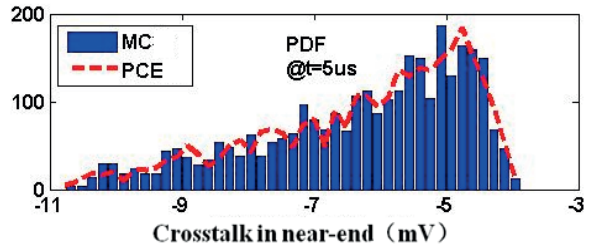

(b) Terminating low impedance

Fig. 14 Comparison of probability density function of crosstalk in near-end based on Legendre-PCE and MC method in time domain
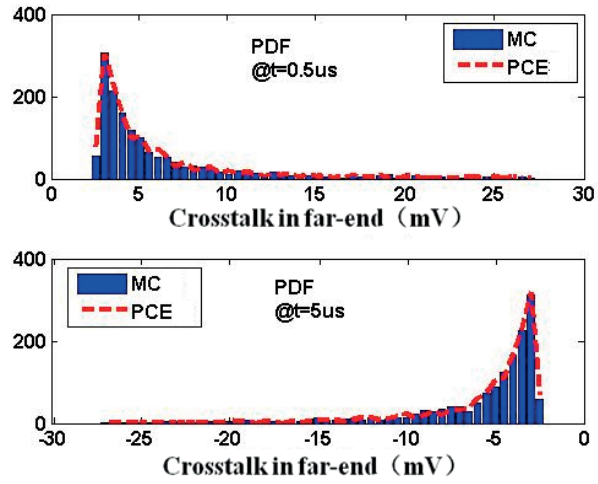

(a) Terminating high impedance

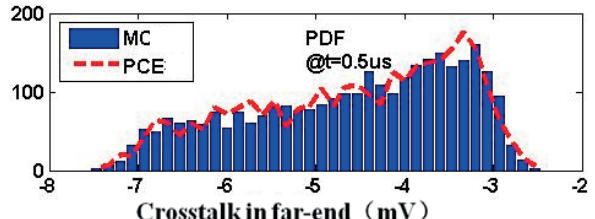

Crosstalk in far-end $(\mathrm{mV})$

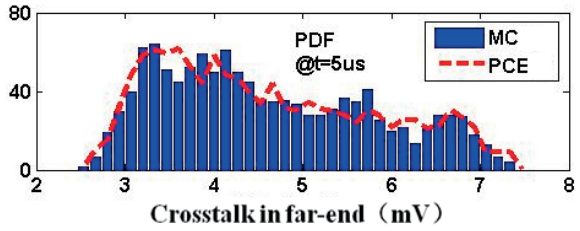

(b) Terminating low impedance

Fig. 15 Comparison of probability density function of crosstalk in far-end based on Legendre-PCE and MC method in time domain 


\section{COMPARISON AND DISCUSSION}

The specific distribution range and the point probability of interference value of naval ships wiring harness at specific frequency can be determined by calculating its probability density function. On the premise of obtaining accuracy results, the PCE method presents much higher efficiency than MC method. Comparison between PCE and MC method with 3000 samples for crosstalk in near-end while terminating high impedance of $1 \mathrm{k} \Omega$ is shown in Table 1 . It can be seen that it only takes 22.32 seconds for Legendre-PCE method while the task takes 34 minutes 21 seconds by adopting the MC method. This proves the high efficiency of the LegendrePCE method.

Tab. 1 Frequency domain comparison between Legendre-PCE and MC method with 3000 samples

\begin{tabular}{|c|c|c|c|}
\hline $\begin{array}{c}\text { Interference } \\
\text { voltage }\end{array}$ & $\begin{array}{c}\text { Terminating } \\
\text { Impedance }\end{array}$ & $\begin{array}{c}\text { PCE } \\
\text { (Legendre) }\end{array}$ & MC (N=3000) \\
\hline Near-end & $\begin{array}{c}\text { High } \\
\text { Impedance } \\
(1 \mathrm{k} \Omega)\end{array}$ & $22.32 \mathrm{~s}$ & $34 \mathrm{~min} 21 \mathrm{~s}$ \\
\hline
\end{tabular}

Based on numerical experiment and analysis in the previous section, the mean value, mean square and varying range in time domain reveals consistency by using LegendrePCE and MC method. Thus, comparison in time domain is performed and is shown in Table 2 between the two methods. As shown in Table 2, the Legendre-PCE method has much shorter time consumption than the MC method. Considering that the results of the two methods are quite close, the Legendre-PCE method is more superior due to its advantage of high-efficiency.

Tab. 2 Time domain comparison between Legendre-PCE and MC method with 3000 samples

\begin{tabular}{|c|c|c|c|}
\hline $\begin{array}{c}\text { Interference } \\
\text { voltage }\end{array}$ & $\begin{array}{c}\text { Terminating } \\
\text { Impedance }\end{array}$ & $\begin{array}{c}\text { PCE } \\
\text { (Legendre) }\end{array}$ & MC (N=3000) \\
\hline Near-end & $\begin{array}{c}\text { High } \\
\text { Impedance } \\
(1 \mathrm{k} \Omega)\end{array}$ & $1 \mathrm{~min} 17 \mathrm{~s}$ & $2 \mathrm{~h} 42 \mathrm{~min} 57 \mathrm{~s}$ \\
\hline
\end{tabular}

Therefore, Legendre-PCE method is adopted to analysis statistical characters of naval ships wiring harness in both time and frequency domain. Results are compared with MC results and comparison show good consistency. Considering that the Legendre-PCE method has better time efficiency than MC method, it is reasonable to adopt this method to applications of the crosstalk in naval ships wiring harness analysis.

\section{CONCLUSIONS}

A high-efficiency analysis method for crosstalk in naval ships wiring harness based on Legendre-PCE is proposed. polynomial chaos expansion method is adopted for analyzing the statistical characters of crosstalk in naval ships wiring harness with random variation of position. Distance among interfering cable, affected cable and GND is synthesized and analyzed in both time domain and frequency domain. Based on the actual 14-cable naval ships wiring harness, detailed distribution parameter, mean value, mean square error, probability density function and reasonable varying range are described in both time domain and frequency domain. The method proposed in this paper, not only has good consistency with the MC method, but also has better time-efficiency than the MC method. Therefore, the PCE method can be applied in the naval ships research field by analyzing electromagnetic compatible issues of wiring harness in order to provide necessary theoretical support and to further guarantee safety.

\section{ACKNOWLEDGEMENTS}

The authors wish to express their gratitude to the National Natural Science Foundation of China (No. 51672103, 51272089), Jilin Jianzhu University, the National Key Technology R\&D Program of the Ministry of Science and Technology of China, the Education Department of Jilin Province of China (20160204069GX), Jilin University, the Fundamental Research Program Foundation of Jilin Province (20150101029JC) and Jilin Provincial Key Laboratory of Architectural Electricity \& Comprehensive Energy Saving for their generous support of this work.

\section{BIBLIOGRAPHY}

1. J.X. Gao, B.M. Manson, P. Kyratsis: Implementation of concurrent engineering in the suppliers to the naval ships industry, Journal of Materials Processing Technology, Vol. 107, no. 1-3, pp.201-208, 2000.

2. Schmidt, K., Tröger, P., Kroll, H., Bünger, T. et al.: Adapted Development Process for Security in Networked Naval ships Systems, SAE Int. J. Passeng. Cars - Electron. Electr. Syst. Vol. 7(2), pp.516-526, 2014.

3. Anna Kochan: Magnetic pulse welding shows potential for naval ships applications, Assembly Automation, Vol. 20, no. 2, pp.129-132, 2000.

4. Jialu Zhang: Topological properties of prime filters in MTLalgebras and fuzzy set representations for MTL-algebras, Fuzzy Sets and Systems, Vol. 178, no.1, pp38-53, 2011.

5. LoVetri Joe, Lapohos Tibor: Explicit upwind schemes for lossy MTL's with linear terminations, IEEE Transactions on Electromagnetic Compatibility, Vol. 39, no. 3, pp. 189200, 1997.

6. Kong, Yongdan, Chu Qingxin, Li Ronglin: High-order unconditionally-stable four-step ADI-FDTD methods and numerical analysis, Progress in Electromagnetics Research, 
Vol. 135, pp. 713-734, 2013.

7. Xiao Fei, Tang Xiaohong, Wang Ling: Stability and numerical dispersion analysis of a 3D hybrid implicitexplicit FDTD method, IEEE Transactions on Antennas and Propagation, Vol. 56, no. 10, pp. 3346-3350, 2008.

8. Cooray F.R., Bird T.S.: Analysis of radiation from two separate circular cylindrical waveguides by the method of moments, Radio Science, Vol. 35, no. 2, pp. 567-577, 2000.

9. Upadhyay Rochan R.: Evaluation of the use of the Chebyshev algorithm with the quadrature method of moments for simulating aerosol dynamics, Journal of Aerosol Science, Vol. 44, pp. 11-23, 2012.

10. Kalantari Nima Khademi, Sen Pradeep: Removing the noise in Monte Carlo rendering with general image denoising algorithms, Computer Graphics Forum, Vol. 32, no. 2, pp. 93-102, 2013.

11. Yeung Jackson H.C., Young Evangeline F.Y., Leong Philip H.W.: A Monte-Carlo floating-point unit for self-validating arithmetic, ACM/SIGDA International Symposium on Field Programmable Gate Arrays - FPGA, pp. 199-207, 2011.

12. Baier Hendrik, Winands Mark H.M.: Monte-Carlo tree search and minimax hybrids with heuristic evaluation functions, Communications in Computer and Information Science, Vol. 504, pp. 45-63, 2014.

13. Sepahvand K., Marburg S., Hardtke H.J.: Stochastic structural modal analysis involving uncertain parameters using generalized polynomial chaos expansion, International Journal of Applied Mechanics, Vol. 3, no. 3, pp. 587-606, 2011.

14. Fagiano Lorenzo, Khammash Mustafa: Simulation of stochastic systems via polynomial chaos expansions and convex optimization, Physical Review E - Statistical, Nonlinear, and Soft Matter Physics, Vol. 86, no. 3, 2012.

15. Hacibekirog G., Cag M., Polatog Y.: The higher order Schwarzian derivative: Its applications for chaotic behavior and new invariant sufficient condition of chaos, Nonlinear Analysis: Real World Applications, Vol. 10, no. 3, pp. 1270$1275,2009$.

16. Nekhamkina Olga, Sheintuch Moshe: Spatially "chaotic" solutions in reaction-convection models and their bifurcations to moving waves, Physical Review E Statistical, Nonlinear, and Soft Matter Physics, Vol. 66, no. 1, pp. 016204/1-016204/5, 2002.

17. Creamer Dennis B.: On using polynomial chaos for modeling uncertainty in acoustic propagation, Journal of the Acoustical Society of America, Vol. 119, no. 4, pp. 1979-1994, 2006.

18. Sandu Corina, Sandu Adrian, Ahmadian Mehdi: Modeling multibody systems with uncertainties. Part II: Numerical applications, Multibody System Dynamics, Vol. 15, no. 3, pp. 241-262, 2006.

19. Guoping Ru, Rong Yu, Yulong Jiang, Gang Ruan: Thermal activation of current in an inhomogeneous Schottky diode with a Gaussian distribution of barrier height, Chinese Physics B, Vol.09, pp. 552-562, 2010.

20. S.M EI shazly: Estimation of Hourly and Daily Global Solar Radiation at Clear Days Using an Approach Based on Modified Version of Gaussian Distribution, Advances in Atmospheric Sciences, Vol. 03, pp. 349-358, 1996.

21. Lei Zhao,Dong Mi,Yeqing Sun: A novel multitarget model of radiation-induced cell killing based on the Gaussian distribution, Journal of Theoretical Biology, Vol. 420, no.7, pp. 135-143, 2017.

\section{CONTACT WITH THE AUTHOR}

Tianhao Wang, Ph.D. e-mail:wangtianhao@jlu.edu.cn

College of Instrumental and Electrical Engineering Jilin University Changchun Jilin 130061 China 